Published Online on 28 September 2016

Proc Indian Natn Sci Acad $\mathbf{8 2}$ No. 4 September 2016 pp. 1167-1170

(C) Printed in India.

DOI: $10.16943 /$ ptinsa/2016/48576

Opinion

\title{
On the need for a National Preprint Repository
}

PRAVEEN CHADDAH*

(Received on 07 August 2016; Revised on 09 August 2016; Accepted on 10 August 2016)

I wish to emphasize theneed and advantages of setting up a national preprint repository as we enhance our research base through newer universities and institutes. There is a widespread impression that submissions to established journals face skeptical referees when these submissions originate from comparatively unknown bylines. The average period between submission and acceptance appears to be longer, and the authors fret about someone else publishing similar work during this period and their losing priority. There is alsoa worry about someone gaining access to the manuscript, and plagiarizing our unpublished work. The well discussed case of ISRO losing priority for discovering water on the moon has been emphasized (Chaddah, 2015a; Chaddah, 2015b; Kochhar, 2010; Lakhotia, 2011) as a striking example of submissions being under review (and being rejected by high impact journals) for just too long. The case of the indicative (as against confirmatory) discovery of high- $\mathrm{T}_{\mathrm{C}}$ superconductive being reported in a comparatively low-visibility journal and thus ensuring priority (and the subsequent Nobel Prize for the discovery) has also been highlighted (Chaddah, 2015a). Clearly one needs to not only plan one's research; one also needs to plan how it should be disseminated.

The immensely popular physics-based preprint archive arXiv.org was set up in 1991 and has changed the way scientific research is now being disseminated (Editorial Nature Physics, 2016). This archive did not exist when Bednorz and Muller found indications of "Possible high- $\mathrm{T}_{C}$ superconductivity" in a multi-phase Ba-La-Cu-O sample. It was, however, immensely popular in the physics (and astrophysics) community when the ISRO communicated their findings to journals. The eventual publication (Sridharan et al.,
2010) was much too late to establish the priority sought to be claimed through the media by the ISRO leaders. Why did Sridharan et al. not take recourse to uploading their manuscript on this preprint archive? We have not had a national debate on the role of disseminating on preprint archives, and allowing postdissemination review. The emphasis on publication in high impact journals has created the impression that pre-publication reviews serve as a validation certificate, in spite of the many cases of retractions from such journals because the data have been found to be falsified. Validation in science is only through post-publication confirmations and tests, and laurels (the Nobel Prize is one example) follow such validation only. Our evaluation systems unfortunately put emphasis on where our work is published, and not on whether it is correct and has impacted other researchers.

One worry that is mentioned in connection with releasing preprints is that there is no protection against the work being used without being cited. This was addressed in a now famous Editorial by the Editor-inChief of APS in September 2005, where he said "Citations should be as complete and up to date as possible and can be drawn from e-print archives as well as peer-reviewed journals." A confirmation of this policy is a correction that acknowledges three papers on the arXiv, even though theywere uploaded after the submission of the original version of the manuscript (Satya et al., 2012)!

I shall describe some examples where the preprint archive has helped Indian groups to claim visibility and priority for their work (See also Chaddah 2016). In early 2008 we had submitted a manuscript to a respected physics journal proposing a

*Author for Correspondence: E-mail: chaddah.praveen@gmail.com 
measurement protocol with a novel thermomagnetic history to bring out the kinetic arrest of a $1^{\text {st }}$ order magnetic transition, to which we gave the acronym CHUF.This was initially rejected without even seeking peer review, and we then uploaded it on arXiv.org (Banerjee et al, 2008), where we highlighted this protocol CHUF in an option provided by arXiv.org, together with the title listing. The manuscript finally appeared in another reputed physics journal after about eight months, and uploading on arXiv.org kept us from wasting time worrying and fretting, and our work progressed with more publications that referred to this preprint that was now in public domain. Sometime later a paper from an established French group appeared in the same journal that had rejected our paper, which had a section describing, utilizing, and praising what they called the "speciallydesigned CHUF measurement protocol". This entire section had no reference and, by implication, they were usurping credit for our work. By the time this French work was submitted we already had many papers utilizing our CHUF measurement protocol, and there were already two papers published in this same respected journal using it and giving us credit. This was clearly a case of referees not following their Editor-in-Chief's dictum on proper citations; this could be attributed to a bias when reviewing a paper from an established group! The journal did publish a correction that, however, is not visible every time the paper is viewed. Fortunately for us, this group had also uploaded their preprint on arXiv.org, and we appealed to their moderators who took appropriate action. Every time a search is done with the author's name on arXiv.org, the apology is visible below the title and the names of the authors (Sarkar et al, 2011). The response from the preprint archive was unbiased. It may only be accidental that arXiv.org has a reputation but no profitability aspects.

Another salient feature of arXiv.org is that authors can modify their uploaded preprints. This can include subsequent results that a journal may not consider major enough for an independent publication. Such changes can be made anytime, and we have used this to include some evolving ideas. Our preprint (Chaddah and Banerjee, 2010) was first uploaded on $19^{\text {th }}$ April 2010. On 14 ${ }^{\text {th }}$ March 2012 we added a new definition of glass formation as "extract specific heat without extracting latent heat", and on 30 $30^{\text {th }}$ July 2012 we added a discussion on how studies on magnetic glass can help understand the physics of formation of structural glass, as distinct from jamming. Such evolution of ideas, over such periods of time, would be difficult to incorporate in a paperpublished in a traditional journal!The preprint archive also provides metrics of downloads, and these reassure us that the subsequent incorporations are also being read.

It must be noted here that all uploads and revisions on arXiv.org remain accessible 'for posterity' and cannot be deleted by the authors. This may be considered as cutting both ways, and I will now describe a case where this has helped another Indian group that is being 'idea-plagiarized' by a Chinese group.In a preprint uploaded on $9^{\text {th }}$ October, 2014 an Indian group (Aggarwal et al, 2014) reported a claim of unconventional superconductivity in the material $\mathrm{Cd}_{3} \mathrm{As}_{2}$. On $2^{\text {nd }}$ January 2015 a Chinese group (Wang et al 2015) reported similar resultson arXiv.org, on the same material. In this they referred to the work of Aggarwal et al, claiming that they saw this after completing their manuscript. This records that they were definitely aware of the Indian work on $2^{\text {nd }}$ January 2015.

Both the groups also submitted manuscripts to the same journal at very different times, where they eventually appeared in the same issue and both were published online on $2^{\text {nd }}$ November 2015 (Aggarwal et al, 2016; Wang et al, 2016a).The Chinese group was not very ethical because it referred to the Indian groups work (Aggarwal et al, 2014) only in 'Note added in proof' even though arXiv.org records show that they were definitely aware of it on $2^{\text {nd }}$ January 2015, if not earlier. These unethical intentions of the Chinese group became obvious when they uploaded another manuscript recently(Wang et al, 2016) claiming "We two groups independently studied $\mathrm{Cd}_{3} \mathrm{As}_{2}$ by using hard point contact spectroscopy and reportedtip induced superconductivity on $\mathrm{Cd}_{3} \mathrm{As}_{2}$ in Nature Materials simultaneously". The reporting was clearly three months apart. The claim of simultaneous reporting by the Chinese group, against the actual simultaneous publishing, is an attempt at unethical usurping of credit. Fortunately, arXiv.org provides hard evidence of priority in reporting, as has been highlighted in the rebuttal on arXiv.org by the Indian group (Gayen et al, 2016). Aggarwal et al have been able to counter this unethical attempt at 'idea-plagiarism' because they 
had released their preprint on arXiv.org in October 2014.

I now discuss the recent 2016 UGC notification on requirements for $\mathrm{Ph}$.D. which states that two paper presentations in conferences/seminars are necessary before the submission of thesis. Most conferences organized in India boldly announce Proceedings will be published by a leading publisher, without mentioning that using the same data for a subsequent journal submission can be considered as self-plagiarism. Also, the organizers ask for manuscripts to be submitted well before the Conference. This makes them available for unauthorized perusal, without the stringent cross-checks that journals routinely use, and present worries of a research scholar losing priority. So, the present scenario for conference presentations raises the spectre of future allegations of self-plagiarism. The 'Academy Policy on Plagiarism' on thePramanawebsite states thatinstances of selfplagiarism will be treated just as seriously as plagiarism from other authors, and such allegations can seriously affect the career.

In this connection, I have proposed (Chaddah 2016) that conference organizers should ask that the submitted manuscripts be uploaded on a preprint archive like arXiv.org rather than the time and effort

\section{References}

Aggarwal L et al. (2014) Unconventional superconductivity at mesoscopic point contacts on the 3D Dirac semimetal $\mathrm{Cd}_{3} \mathrm{As}_{2}$ arXiv: 1410.2072

Aggarwal L et al. (2016) Unconventional superconductivity at mesoscopic point contacts on the 3D Dirac semimetal $\mathrm{Cd}_{3} \mathrm{As}_{2}$. Nat Mat 15 32-37

Banerjee A et al. (2008) Conversion of glassy antiferromagneticinsulating phase to equilibrium ferromagnetic-metallic phase by devitrification and recrystallization in $\mathrm{Al}$ substituted $\mathrm{Pr}_{0.5} \mathrm{Ca}_{0.5} \mathrm{MnO}_{3}$ arXiv: 0805.1514

Bednorz J G and Müller K A (1986) Possible High-T ${ }_{C}$ superconductivity in the Ba-La-Cu-O system Z Phys $\mathbf{B 6 4}$ 189-193

Chaddah P and Banerjee A (2010) Magnetic Glass formed by kinetic arrest of first order phase transitions arXiv: 1004.3116

Chaddah P (2016) The importance of a preprint repository Current Science 111 (in press) consuming process of bringing out a 'proceedings'. It is interesting that even Nature Research Journals are looking forward to "allow authors to submit a paper by simply entering its arXiv number" (Editorial Nature Physics, 2016). Nature Research Journals has been conservative; Physical Review has been allowing this for some years already. Many journals also allow uploading of modified manuscripts at intermediate stages of refereeing whereas Nature presently has an embargo at this level. The widespread use of arXiv.org by physicists is driving changes in the policies of traditional journals. It is also influencing other disciplines. BioRxiv was launched in November 2013 by the nonprofit Cold Spring Harbor Laboratory as a biologists' version of arXiv, and has become quite popular.Paul Ginsparg, the physicist who started arXiv in 1991, serves on bioRxiv's advisory board.

Since arXiv.org has changed the way research output is disseminated, with authors deciding when they are ready to share them, many more preprint servers have been set up. These cover many disciplines, including social science and humanities. The Academy could identify such preferred archives for various branches of sciences. Or, we could work towards setting up a National Preprint Repository covering all disciplines.

Chaddah P (2015a) Lessons on Impact Factorfrom the 'DBTand DSTOpen Access Policy’ Proc INSA 81 553-555

Chaddah P (2015b) Do we lack courage in research? Current Science 108 313-313

Editorial (2016) Keep posting Nature Physics 12719

Gayen S et al. (2016) Comment on "Tip induced unconventional superconductivity on Weyl semimetal TaAs" arXiv: 1607.01405

Kochhar R (2010) 'Engineering vis-a-vis science': India's missed discovery of water on the moon Current Science 98 15491550

Lakhotia S C (2011) 'Impact factor' and 'we also ran' syndrome Current Science 99411

Sarkar T, Pralong V and Raveau B (2011) Formation of magnetic glass in calcium doped $\mathrm{YBaCo}_{2} \mathrm{O}_{5.5}$ cobaltitesarXiv: 1106.6253

Satya A T et al. (2012) Erratum: Pressure-induced metallization of $\mathrm{BaMn}_{2} \mathrm{As}_{2}$ [Phys Rev B84 180515(R) (2011)] Phys Rev 85019901 
SridharanR et al (2010) 'Direct' evidence for water $\left(\mathrm{H}_{2} \mathrm{O}\right)$ in the sunlit lunar ambience from CHACE on MIP of Chandrayaan I, Planetary and Space Science 58 947-950

Wang H et al. (2015) Observation of superconductivity induced by a point contact on $3 \mathrm{D}$ Dirac semimetal $\mathrm{Cd}_{3} \mathrm{As}_{2}$ crystals arXiv: 1501.00418
Wang H et al. (2016a) Observation of superconductivity induced by a point contact on 3D Dirac semimetal $\mathrm{Cd}_{3} \mathrm{As}_{2}$ crystals Nat Mat 15 38-42

Wang H et al. (2016b) Reply to Comment on "Tip induced unconventional superconductivity on Weyl semimetal TaAs" arXiv: 1607.02886. 